\title{
TRAUMATISMO CRANIOENCEFÁLICO NO MUNICÍPIO DE FORTALEZA
}

Josi Andrade da Silva ${ }^{1}$, Adriano Rodrigues de Souza $^{1}$, Aline Rodrigues Feitoza' ${ }^{1}$, Tatiana de Medeiros Colletti Cavalcante ${ }^{1}$

Objetivo: traçar o perfil epidemiológico dos pacientes com diagnóstico de traumatismo cranioencefálico (TCE) atendidos em um hospital de referência em trauma no município de Fortaleza-CE. Metodologia: trata-se de um estudo descritivo, quantitativo e documental com 722 prontuários de vitimas de TCE. Resultados: a pesquisa mostrou maior incidência de casos no gênero masculino, com idades entre 21 e 30 anos, provenientes do interior do estado, cujo maior número de internamentos aconteceram no final de semana. Quanto à evolução dos casos, $84 \%$ tiveram alta, enquanto $16 \%$ foram a óbito. Conclusão: diante dos resultados obtidos, concluímos que o conhecimento deles se torna uma importante ferramenta de implementação de medidas de intervenção com vistas à prevenção e à redução dessas lesões.

Descritores: Traumatismos craniocerebrais, Epidemiologia, Traumatismos encefálicos.

\section{TRAUMATIC BRAIN INJURY IN THE MUNICIPALITY OF FORTALEZA}

Objective: to trace the epidemiological profile of patients with diagnosis of traumatic brain injury (TBI) treated at a referred hospital for trauma in Fortaleza-CE. Methodology: This is a descriptive, quantitative and documentary study with 722 medical records of victims of TBI. Results: Research has shown a higher incidence of cases in males aged 21 to 30 years from the countryside of the state, where the highest number of hospitalizations occurred over the weekend. As for the evolution of the cases, $84 \%$ were discharged while $16 \%$ died. Conclusion: based on the results obtained, we conclude that their knowledge becomes an important tool for implementing the intervention measures aimed at preventing and reducing these injuries.

Descriptors: Craniocerebral trauma, Epidemiology, Brain injury.

\section{LA LESIÓN CEREBRAL TRAUMÁTICA EN EL MUNICIPIO DE FORTALEZA}

Objetivo: trazar el perfil epidemiológico de los pacientes con lesión cerebral traumática (LCT) tratado en un hospital de referencia para el trauma en Fortaleza-CE. Metodología: Se trata de un estudio descriptivo, cuantitativo y documental de 722 historias clínicas de las víctimas de LCT. Resultados: La investigación ha demostrado una mayor incidencia de casos en varones de 21 a 30 años desde el interior del estado, cuyo mayor número de hospitalizaciones ocurrido durante el fin de semana. A medida que la evolución de los casos fueron dados de alta $84 \%$ mientras que el $16 \%$ murió. Conclusión: los resultados obtenidos, llegamos a la conclusión de que su conocimiento se convierte en una herramienta importante para la aplicación de las medidas de intervención dirigidas a la prevención y la reducción de estas lesiones.

Descriptores: Traumatismo craneoencefálico, Epidemiología, Lesión cerebral. 


\section{INTRODUÇÃO}

O traumatismo cranioencefálico (TCE) é uma lesão do parênquima cerebral de origem não degenerativa ou congênita, provocada por uma força física externa, como um choque local ou movimentos repetidos decorrentes de impactos únicos ou múltiplos, resultando em compressão, expansão, aceleração, desaceleração e rotação do cérebro dentro do crânio. Essa lesão pode provocar alterações do nível de consciência e produzir deficiências dos desempenhos cognitivos, comportamental, emocional ou físico. As deficiências de ordem física tornam-se aparentes na fase subaguda e crônica, tais como distúrbios musculoesqueléticos, cardiovasculares, endócrinos, alterações no trato gastrointestinal e trato urinário, além de comprometimento no sensório-motor e na dinâmica respiratória ${ }^{(1,2)}$.

O TCE pode causar lesões variáveis e desencadear uma cascata de sequelas com dano celular. As lesões podem ser decorrentes de trauma aberto ou fechado. classificadas em focais ou difusas. As lesões difusas são quase sempre microscópicas e estão associadas à disfunção generalizada do parênquima cerebral. Já as lesões focais, geralmente, são macroscópicas e limitadas à determinada área, como consequência de um trauma localizado; os déficits decorrentes desse trauma são semelhantes aos observados em pacientes com Acidente Vascular Encefálico(1)

Quanto ao grau da lesão, pode ser classificada como leve, moderada e grave. Entre os índices de gravidade estão a profundidade e a duração do coma avaliado pela escala de Glasgow. Segundo essa escala, pacientes com pontuação entre 13 a 15 pontos na escala configuram um TCE leve, de 9 a 12 moderado e entre 3 a 8 grave ${ }^{(3)}$.

O trauma leve é definido como déficit neurológico transitório, provocando alteração do nível de consciência e amnésia retrógada por um breve período de tempo. No entanto, esses pacientes podem apresentar sequelas tardias que se assemelham às do TCE grave, apesar de serem pouco frequentes. No moderado, observa-se perda da consciência por um período superior a 5 minutos e/ ou déficit neurológico focal; enquanto que, no grave, há alteração significativa do nível de consciência, avaliado pela gravidade da pontuação na escala de coma de Glasgow $^{(2)}$

No Brasil, a epidemiologia do trauma cranioencefálico é estimada em meio milhão de hospitalizações anualmente ${ }^{(4)}$. O Ceará, no ano de 2013, atendeu 6.480 pacientes com diagnóstico de traumatismo intracraniano. Fortaleza foi responsável por 49\% (3.160) dessas internações ${ }^{(5)}$.

Uma pesquisa realizada entre janeiro a dezembro de 2006 no Instituto Dr. José Frota (IJF) indicou que 60\% (725) dos TCE's são provocados por acidente de trânsito(6). Entre esses, cerca de $80 \%$ dos pacientes são classificados como trauma leve, $10 \%$ como moderados e $10 \%$ são quadros classificados como grave ${ }^{(7)}$. Os grupos dos traumas grave e moderado necessitam de constante avaliação médica e cuidados intensivos na unidade de terapia intensiva (UTI), onde recebem cuidados especializados com o intuito de manter os parâmetros hemodinâmicos e evitar lesões secundárias ao trauma.

O presente estudo surgiu a partir do interesse em conhecer quantitativamente as vitimas de trauma cranioencefálico atendidas no hospital de referência em trauma no município de Fortaleza. A partir disso, surgiram os seguintes questionamentos: quem são os sujeitos vítimas de TCE? Qual a quantidade de atendimento desse tipo de trauma no IJF no ano de 2013? Quais as medidas implementadas pelo governo, visando a redução dessas lesões?

Ao responder a todos esses questionamentos, estaremos contribuindo para que se conheça o perfil das vitimas desse trauma na capital cearense, visando o gerenciamento dos casos dentro e fora da instituição alvo da pesquisa. Portanto, temos como objetivo traçar o perfil dos pacientes com TCE internados em um hospital de referência em trauma na cidade de Fortaleza.

\section{METODOLOGIA}

Pesquisa quantitativa de natureza descritiva e de segmento transversal; descreve uma situação ou fenômeno em um momento não definido, apenas representado pela presença de uma doença ou transtorno, 
e cuja exposição ao fator ou causa está presente ao efeito no intervalo de tempo analisado. Esse tipo de pesquisa apresenta-se como um corte instantâneo de uma determinada população por meio de uma amostragem, na qual se examina, nos integrantes, a presença ou ausência de exposição e a presença ou ausência do efeito/doença ${ }^{(8)}$.

O estudo foi realizado em um hospital do município de Fortaleza, considerado o maior hospital de urgência e emergência do estado do Ceará, sendo referência no atendimento ao politraumatizado. O hospital tem capacidade para 461 leitos, dentre eles, 33 leitos de UTI, onde dispõe de uma UTI pediátrica com sete leitos e três unidades destinadas ao cuidado de adultos com um total de 26 leitos. Realiza anualmente em torno de 146.989 atendimentos ${ }^{(9)}$.

A população do estudo foi composta por 722 prontuários de pacientes com diagnóstico de TCE internados no hospital, no período de janeiro a dezembro de 2013. Os dados foram coletados no período de maio a dezembro de 2014, tendo como variáveis de coleta: sexo, idade, procedência, dia da semana da ocorrência, número de óbitos e diagnóstico segundo Classificação Internacional de Doenças e Agravos a Saúde 10 ${ }^{a}$ edição (CID-10). Os dados coletados foram agrupados e inseridos no programa EPIDATA versão no 10 analisados no Stata versão $n$ ㅇ 10, tratados estatisticamente e apresentados em forma de tabelas.

A pesquisa foi aprovada no comitê de ética da instituição envolvida, sob o parecer de número 625.779/2014.

\section{RESULTADOS}

O presente estudo analisou 722 prontuários de pacientes com diagnóstico de TCE, nos quais predominou o gênero masculino, com 591 (82\%) registros. A idade das vítimas concentrou-se entre $21-30$ anos, com 158 (22\%), seguida da faixa etária de 11-20 anos, com 116 (16\%) casos. Ao avaliar a procedência das internações, temos que 374 (52\%) dos casos são oriundos das cidades interioranas, enquanto que 341 (47\%) dos internados eram provenientes da capital do estado. O dia da semana com maior número de internações por TCE foi domingo, com 224 (31\%) atendimentos, seguidos do sábado, com 159 (22\%) (Tabela 1).

A respeito das unidades para onde os pacientes são direcionados após sua avaliação, 419 (58\%) foram para as unidades de neurocirurgia, 167 (23\%) para as unidades de clínica médico-cirúrgicas e toracovascular, 62 (9\%) para as unidades de traumatologia, enquanto 35 (5\%) foram para as UTI's. Os demais estão na tabela 1. Quanto à evolução dos casos, 606 (84\%) receberam alta e, infelizmente, 116 (16\%) vítimas foram a óbito (Tabela 1 ).
Tabela 1: Distribuição dos Traumatismos Cranioencefálicos (TCE), atendidos no Hospital de Referência em trauma de Fortaleza em 2013.

\begin{tabular}{|c|c|c|}
\hline VARIÁVEIS ( $\mathrm{N}=722)$ & № & $\%$ \\
\hline \multicolumn{3}{|l|}{ SEXO } \\
\hline Masculino & 591 & 82 \\
\hline Feminino & 131 & 18 \\
\hline \multicolumn{3}{|l|}{ FAIXA ETÁRIA (ANOS) } \\
\hline $1-10$ & 77 & 11 \\
\hline $11-20$ & 116 & 16 \\
\hline $21-30$ & 158 & 22 \\
\hline $31-40$ & 112 & 15 \\
\hline $41-50$ & 92 & 13 \\
\hline $51-60$ & 65 & 9 \\
\hline$>60$ anos & 102 & 14 \\
\hline \multicolumn{3}{|l|}{ PROCEDÊNCIA } \\
\hline Capital & 341 & 47 \\
\hline Outros municipios do estado & 374 & 52 \\
\hline Sem informação & 7 & 1 \\
\hline \multicolumn{3}{|l|}{ DIA DA SEMANA } \\
\hline Domingo & 224 & 31 \\
\hline Sábado & 159 & 22 \\
\hline Sexta-feira & 115 & 16 \\
\hline Segunda-feira & 87 & 12 \\
\hline Quarta-feira & 58 & 8 \\
\hline Quinta-feira & 43 & 6 \\
\hline Terça-feira & 36 & 5 \\
\hline \multicolumn{3}{|l|}{ UNIDADES DE INTERNAÇÃO } \\
\hline $\begin{array}{l}\text { Unidades neurologia/ } \\
\text { neurocirurgia }\end{array}$ & 419 & 58 \\
\hline $\begin{array}{l}\text { Clínica médica-cirúrgicas/ } \\
\text { toracovascular }\end{array}$ & 167 & 23 \\
\hline Unidades traumatológicas & 62 & 9 \\
\hline UTI'S I, II, III e IV & 35 & 5 \\
\hline Plástica/BMF & 25 & 3 \\
\hline Cirurgia geral & 14 & 2 \\
\hline \multicolumn{3}{|l|}{ EVOLUÇÃO DOS CASOS } \\
\hline Alta & 606 & 84 \\
\hline Óbito & 116 & 16 \\
\hline
\end{tabular}

Fonte: prontuários

Quanto à classificação das lesões conforme CID-10, 181 (25\%) casos foram classificados sob o código S06.9, o qual é descrito como Traumatismo intracraniano não- 
especificado. A segunda maior classificação foi o S06.8. Outros traumatismos intracranianos com 174 (24\%) casos, os demais CID's estão descritos na Tabela 2.

Tabela 2: Distribuição dos TCE's, atendidos no Hospital de Referência em trauma de Fortaleza em 2013 conforme CID-10

\begin{tabular}{|c|c|c|c|}
\hline CID & DESCRIÇÃO NA CID & $\mathbf{N}$ & $\%$ \\
\hline S06.9 & $\begin{array}{l}\text { Traumatismo intracraniano } \\
\text { não especificado }\end{array}$ & 181 & 25 \\
\hline S06.8 & $\begin{array}{l}\text { Outros traumatismos } \\
\text { intracranianos }\end{array}$ & 174 & 24 \\
\hline S06.2 & Traumatismo cerebral difuso & 166 & 23 \\
\hline S06.1 & Edema cerebral traumático & 101 & 14 \\
\hline S06.4 & Hemorragia epidural & 29 & 4 \\
\hline s06.5 & $\begin{array}{l}\text { Hemorragia subdural devido } \\
\text { a traumatismo }\end{array}$ & 22 & 3 \\
\hline s06.7 & $\begin{array}{l}\text { Traumatismo intracraniano com } \\
\text { coma prolongado }\end{array}$ & 14 & 2 \\
\hline S06.3 & Traumatismo cerebral focal & 7 & 1 \\
\hline S02.0 & Fratura de abóboda do crânio & 14 & 2 \\
\hline S02.7 & $\begin{array}{l}\text { Fraturas múltiplas envolvendo os } \\
\text { ossos do crânio e da face }\end{array}$ & 7 & 1 \\
\hline \multirow[t]{2}{*}{ S09.9 } & $\begin{array}{l}\text { Traumatismo não especificado } \\
\text { da cabeça }\end{array}$ & 7 & 1 \\
\hline & Total & 722 & 100 \\
\hline
\end{tabular}

\section{DISCUSSÃO}

No presente estudo, $82 \%$ das vítimas pertencem ao sexo masculino, 22\% têm entre 21 e 30 anos de idade. Pesquisa semelhante foi apresentada por Viégas (2013), na qual $88 \%$ eram homens e $32 \%$ tinham idade entre 20 30 anos $^{(10)}$. Diferente do Brasil, nos Estados Unidos, a faixa etária mais acometida pelo TCE são os idosos acima de 75 anos, vítimas de quedas, segundo relatório publicado pelo Centers for Disease Control and Prevention (CDC) em 2015(11). A tendência de gênero e idade no Brasil pode estar relacionada ao estilo de vida, à busca por desafios, à pouca experiência na pilotagem de veículos, ao baixo grau de escolaridade, bem como, à dificuldade em adquirir a Carteira Nacional de Habilitação $(\mathrm{CNH})^{(6)}$. O governo do estado do Ceará tem tentado mudar essa realidade por meio da educação no trânsito e aquisição da primeira $\mathrm{CNH}$ gratuitamente para os condutores de baixa renda. No ano de 2009, por intermédio do Departamento Estadual de Trânsito (DETRAN), o governo do estado instituiu o Programa Popular de Formação, Educação, Qualificação e Habilitação Profissional de Condutores de Veículos
Automotores, com a publicação da Lei №14.288-A, de 06 de janeiro de $2009^{(12)}$

A respeito da procedência, $52 \%$ dos pacientes com diagnóstico de TCE são provenientes do interior do estado. Vale salientar que o hospital do estudo é referência em trauma para o estado do Ceará, atendendo à demanda de maior complexidade do estado.

Quanto à destinação dos pacientes, após seu primeiro atendimento e posterior realização de exames, conforme protocolo da instituição, são encaminhados às unidades de internação. Esse estudo mostrou que $58 \%$ das internações são realizadas nas unidades neurocirúrgicas, o que pode estar ligado à gravidade do trauma, pois, a maioria dos TCE's são classificados como leves( ${ }^{(7)}$

Outra importante observação é o percentual de pessoas encaminhadas para os leitos de UTI. Essa pesquisa mostrou que apenas $5 \%$ dos casos pesquisados foram internados na UTI, o que pode estar ligado ao número reduzido desse tipo de leito no estado. A portaria de $n^{\circ} 1101 / G M$ de 2002 regulamenta que, para cada 10.000 habitantes, deve-se ter de 1 a 3 leitos de UTI(13). No Ceará, segundo dados do CREMEB, os leitos de UTI disponíveis para os usuários do Sistema Único de Saúde (SUS) estão em torno de 0,77, indicando um índice abaixo do preconizado pelo Ministério da Saúde ${ }^{(14)}$

Essa pesquisa apontou que o dia da semana com maior número de atendimentos foi domingo, com $31 \%$, seguidos do sábado com $22 \%$, sexta-feira $16 \%$ e os demais dias somam $31 \%$ dos internamentos. Um estudo semelhante, realizado no município de Petrolina, no estado de Pernambuco, também apontou domingo como o dia com o maior número de internamentos por TCE(15).

Quanto ao desfecho, 84\% receberam alta, enquanto 16\% dos TCE's foram a óbito. O estudo de Moura (2011) aponta uma evolução maior dos casos de alta 88,12\% e menor no número de óbitos de 7,92\% por essa lesão.

Em relação à classificação dos TCE's conforme CID-10(16), o grupo S06 Traumatismo intracraniano foi o mais prevalente, com 96\% dos registros; estratificando esse grupo, os três maiores diagnósticos foram Traumatismo intracraniano não especificado, com $25 \%$, seguido por Outros traumatismos Intracranianos, com 24\%, e Traumatismo cerebral difuso, com 23\%. Uma pesquisa realizada por Moll (2015) apontou dois principais grupos de diagnósticos relacionados ao TCE, conforme CID-10, o S06 - Traumatismo Intracraniano e SO7 - Esmagamento de Cabeça ${ }^{(17)}$. Semelhantemente ao nosso estudo, o principal grupo de diagnóstico da pesquisa de Moll, foi S06 Traumatismo intracraniano e, o maior subgrupo, o traumatismo intracraniano não especificado com 17,1\%. No entanto, no nosso estudo, esse percentual apresentou-se 
mais elevado. Segundo a autora, os referidos diagnósticos são inespecíficos, o que, sob o ponto de vista epidemiológico, dificulta a interpretação da magnitude e gravidade dos $\operatorname{casos}^{(17)}$

Este estudo apresenta limitações quanto ao tamanho de sua amostra, visto que, foi realizado em apenas um dos hospitais da rede de atendimento às urgências e emergências com foco no trauma do município de Fortaleza.

\section{CONCLUSÃO}

Concluímos que a maioria das vítimas de TCE são adultos jovens do sexo masculino, provenientes do interior do estado. Os internamentos ocorreram principalmente no final de semana. A maioria dos pacientes foram internados nas unidades neurocirúrgicas, obtendo alta hospitalar. Vale salientar que o conhecimento do perfil epidemiológico das vítimas de TCE é uma importante ferramenta de medida de intervenção, com vistas à prevenção e redução dessas lesões. Além disso, faz-se necessário uma maior disposição/criação de leitos de UTI no estado para atender a regulamentação do Ministério da Saúde e, assim, proporcionar melhor assistência a esses pacientes.

REFERÊNCIAS

1. Almeida TLT; Falkenburg L; Nascimento RZR; Reis CA; Sales VC; Pedroso TD; et al. Associação Brasileira de Medicina Física e Reabilitação. Traumatismo Cranioencefálico: Reabilitação. Projeto Diretrizes. [Internet] 2012. Disponivel em: <http://www.projetodiretrizes.org.br/projeto_ diretrizes2013/traumatismo_cranioencefalico_reabilitacao.pdf> Acesso em: 09/01/2015.

2. Ministério da Saúde(BR). Diretrizes de atenção à pessoa com traumatismo craniencefálico. [Internet] 2013. Disponivel em: <http://portalsaude.saude. gov.br/portalsaude/arquivos/pdf/2013/Jan/03/DIRETRIZES_TCE.pdf> Acesso em: 09/01/2015.

3. Díaz AP. Transtornos psiquiátricos e qualidade de vida após traumatismo crânio-encefálico grave - um estudo prospectivo [dissertação]. Florianópolis:Universidade Federal de Santa Catarina; 2011. Disponivel em: <https://repositorio.ufsc.br/handle/123456789/95087 >Acesso em: 09/01/2014

4. Maia BG; De Paula FRP; Cotta GD; Lima Cota MAL; Públio PG; Oliveira $\mathrm{H}$ de; et al. Perfil Clínico-Epidemiológico das Ocorrências de Traumatismo Cranioencefálico. Rev Neurociênc. [Internet] 2013. 21(1):43-52. Disponivel em: http://www.revistaneurociencias.com.br/edicoes/2013/RN2101/ original2101/786original.pdf

5. Ministério da saúde [BR]. DATASUS. Informações de saúde. Morbidade hospitalar. [Internet] 2013. Disponivel em: <http://tabnet.datasus.gov.br/ cgi/tabcgi.exe?sih/cnv/nice.def> Acesso em: 27/11/2015.

6. Barbosa IL, Andrade LM, Caetano JA, Lima MA, Vieira LJES, Lira SVG. Fatores desencadeantes ao trauma cranioencefálico em um hospital municipal. Rev.Bai.Sau.Púb. [Internet] 2010. 34(2):240-253. Disponivel em: <http://inseer.ibict.br/rbsp/index.php/rbsp/article/viewFile/31/31>. Acesso em: 01/07/2015.

7. Gentile JKA; Himuro HS; Rojas SSO; Veiga VC; Amaya, LEC; Carvalho JC. Condutas no paciente com trauma cranioencefálico. Rev Bras Clin Med. [Internet] 2011. 1(9):74-82. Disponivel em: http://formsus.datasus.gov.br/ novoimgarq/15106/2268662_109706.pdf> Acesso em: 12/01/2015.

8. Hochman B; Nahas FX; Oliveira filho RS; Ferreira LM. Desenhos de pesquisa. Acta Cirúrgica Brasileira [Internet] 2005. 20(2)aO2. Disponivel em: <http://www.scielo.br/pdf/acb/v20s2/v20s2a02.pdf> Acesso em 23/12/2015

9. Fortaleza - Ceará. Histórico. Instituto Dr. José Frota. [Internet] 2014; Disponivel em: <http://www.fortaleza.ce.gov.br/ijf/historico>Acesso em: $08 / 01 / 2015$
10. Viégas MLC; Pereira ELR; Targino AA; Furtado VG; Rodrigues DB Traumatismo cranioencefálico em um hospital de referência em trauma no estado do Pará, Brasil: prevalência das vitimas quanto a gênero, faixa etária. mecanismo de trauma e óbito. ArqBrasNeurocir. [Internet] 2013. 1(32):15-8. Disponivel em: http://files.bvs.br/upload/S/0103-5355/2013/v32n]/a3620. pdf. Acesso em: 05/07/2016.

11. Centers for Disease Control and Prevention-CDC. Report to Congress on Traumatic Brain Injury in the United States: Epidemiology and Rehabilitation. National Center for Injury Prevention and Control: Division of Unintentional Injury Prevention. [Internet] 2015. Atlanta, GA. Disponivel em: $\quad$ http://www.cdc.gov/traumaticbraininjury/pdf/tbi_report_to_ congress_epi_and_rehab-a.pdf> Acesso em: 05/07/2016.

12. Ceará. Lei no LEI №14.288-A, de 06 de janeiro de 2009. Lei №14.288-a: Fortaleza, CEARÁ: Casa Civil, 27 jan. 2009. Disponivel em: <http://imagens. seplag.ce.gov.br/PDF/20090127/do20090127p01.pdf>. Acesso em: 05/07/2016

13. Ministério da Saúde (Brasil). Portaria 1102 de 2002. Estabelece os parâmetros de cobertura assistencial no âmbito do Sistema Único de Saúde. Disponível em: <http://www.saude.mg.gov.br/images/documentos/ PORTARIA_1101.pdf> Acesso em 10/08/2016.

14. Conselho Regional de Medicina do Estado da Bahia [homepage na internet]. 5065 municipios brasileiros não possuem leitos de UTI, aponta estudo do Conselho Federal de Medicina. Disponivel em: <http://www. cremeb.org.br/index.php/noticias/86-das-cidades-brasileiras-naopossuem-nenhum-leito-publico-de-uti/> Acesso em: 10/08/2016.

15. Moura JC; Rangel BLR; Creôncio SCE. Perfil clínico-epidemiológico de traumatismo cranioencefálico do Hospital de Urgências e Traumas no municipio de Petrolina, estado de Pernambuco. Arq. bras. Neurocir. 2011 30(3)a2709. Disponivel em: <http://files.bvs.br/upload/S/0103-5355/2011/ v30n3/a2709.pdf>. Acesso em 05/07/2016.

16. Organização Mundial de Saúde - OMS. Classificação Internacional de Doenças. 10å edição. CID-10. Disponivel em: http://www.datasus.gov.br/ cid10/V2008/WebHelp/s00_s09.htm>. Acesso em: 06/07/2016.

17. Moll AVS. Perfil do atendimento dos pacientes com traumatismo cra nioencefálico nos hospitais de urgência e emergência sob a gestão da Secretaria de Estado de Saúde do Rio de Janeiro [dissertação]. Rio de Janeiro:Escola Nacional de Saúde Pública Sergio Arouca;2015. Disponivel em: <http://bases.bireme.br/cgi-bin/wxislind.exe/iah/online/ ?IsisScrip $\mathrm{t}=\mathrm{iah} / \mathrm{iah} . \mathrm{xis} \& \mathrm{src}=\mathrm{google}$ base=LILACSElang=p\&nextAction=lnk\&exprSea rch=7824708indexSearch=ID>. Acesso em: 06/07/2016. 